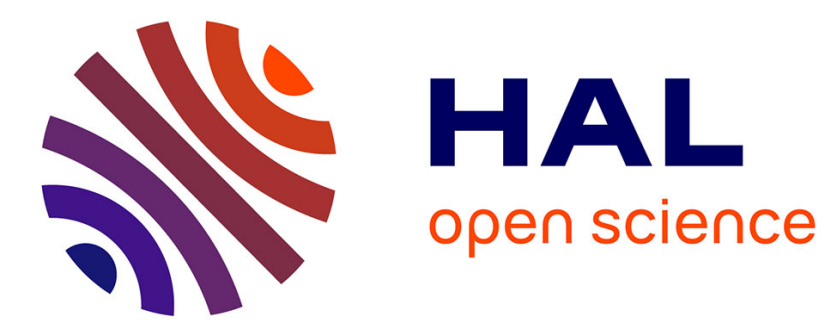

\title{
Dynamical system model of decision making and propagation
}

\author{
Jacques Demongeot, Vitaly Volpert
}

\section{To cite this version:}

Jacques Demongeot, Vitaly Volpert. Dynamical system model of decision making and propagation. Journal of Biological Systems, 2015，10.1142/S0218339015500187 . hal-01237695

\section{HAL Id: hal-01237695 \\ https://hal.science/hal-01237695}

Submitted on 8 Dec 2015

HAL is a multi-disciplinary open access archive for the deposit and dissemination of scientific research documents, whether they are published or not. The documents may come from teaching and research institutions in France or abroad, or from public or private research centers.
L'archive ouverte pluridisciplinaire HAL, est destinée au dépôt et à la diffusion de documents scientifiques de niveau recherche, publiés ou non, émanant des établissements d'enseignement et de recherche français ou étrangers, des laboratoires publics ou privés. 


\title{
Dynamical System Model of Decision Making and Propagation
}

\author{
J. Demongeot ${ }^{1}$, V. Volpert ${ }^{2}$ \\ ${ }^{1}$ Laboratory AGIM, FRE CNRS 3405, University J. Fourier of Grenoble, Faculty of Medicine \\ 38700 La Tronche, France \\ ${ }^{2}$ Institut Camille Jordan, UMR 5208 CNRS, University Lyon 1, 69622 Villeurbanne, France \\ Corresponding author. E-mail: volpert@math.univ-lyon1.fr
}

\begin{abstract}
Individual decision making is described as a bistable dynamical system. It can be influenced by the environment represented by other individuals, public opinion, all kinds of visual, oral and other information. We will study how the interaction of the individual decision making with the environment results in various patterns of decision making in the society.
\end{abstract}

Key words: individual decision making, decision propagation, travelling waves, pattern of decisions

\section{Decision theory}

The theory of decision making intensively develops in relation with numerous applications in business, economy, politics, medicine and so on [1], [2]. It deals with goal-directed behavior in the presence of options [3]. It appeared in the middle of the last century at the intersection of many different sciences, economy, psychology, statistics, neuroscience, and others. However already in the end of the XVIII-th century Condorcet described the process of decision making by a group of individuals suggesting several stages of this process [4]. Modern decision theory also considers decision making as a stage process. In sequential models there are several consecutive steps of decision making [5], [6]. In [7] the following stages are suggested: identification of the problem, obtaining necessary information, production of possible solutions, evaluation of such solutions, selection of a strategy for performance. In non-sequential models, the stages can have a more complex relation including their cycling [8].

The process of decision making consists in comparing two or several options to choose the best of them according to some criteria. It implies some order in the set of decisions and the possibility to compare them. For any two of them, decisions can be considered as better, worth, or equal to each other. This relation should possess the property of transitivity. Decision making is often associated with its utility in purpose of its maximization 
or satisfactoriness [9]. In individual decision making, the standard representation of the problem is based on decision matrices where alternative choices are related to the states of nature, that is to unknown extraneous factors [3]. Since the state of nature may not be completely known, there is a scale of knowledge situation in decision making: certainty, risk, uncertainty, ignorance [10].

Recent works on the theories of reasoning, judgment and decision making can be found in [11]. Evolutionary aspects of decision making are discussed in [12]. Decision making under constraints is studied in [13]. Mathematical theories of decision making are based on the game theory [14], [15], [16] probability and, more generally, uncertainty theories [17], [18], and on linear programming and optimization [19].

In this work we will consider decision making process as dynamical system where individual decision making is influenced by his/her (=hir) environment. For the process of individual decision making we will suppose that the problem is identified and its solution consists of two alternatives. The individual possesses all or partial information necessary for decision making, and the process of decision making consists of evaluation of possible decisions with respect to their utility. Therefore we consider the standard stage approach to decision making. However we concentrate mainly only on one of these steps, evaluation of alternative decisions and we describe it as a bistable dynamical system. This means that decision making process can be characterized by some continuous variables which measure the likelihood to adopt one of possible decisions. If there are two stable decisions, then we will describe this process with two variables $p_{1}$ and $p_{2}$ which we call the willingness to adopt decision 1 and the willingness to adopt decision 2 . These two variables compete with each other, and if one of them is sufficiently large and another one is sufficiently small, then the corresponding decision will be adopted.

Individual decision making can be influenced by the environment which we understand in a large sense. This can be other individuals, public opinion, all kinds of visual, oral and other information got by the individual which can influence hir decision. This can also be even hir own physical conditions. The main goal of this work is to analyze how the interaction of the individual decision making with the environment results in various patterns of decision making in the society. In the next section we will introduce the model of individual decision making, and in Section 3 we will study the interaction of individual decision making with the environment. We will study how the groups of decided individuals expand in space, and whether these groups are homogeneous with respect to the adopted decision or their decisions can be different.

\section{Individual decision making}

\subsection{Basic model of decision making}

Consider an individual who is supposed to make a choice between two decisions. We introduce some variables which characterize the individual with respect to these decisions. We will call them the willingness to adopt decision 1 and the willingness to adopt decision 2 . 
The corresponding variables are denoted by $p_{1}$ and $p_{2}$ respectively. If $p_{1}>p_{2}$, then it is more likely that the individual will adopt decision 1 and vice versa. However we need to describe more precisely the process of decision making.

The process of decision making is a time evolution of the variables $p_{1}$ and $p_{2}$. They have some initial values which depend on the personal experience of the individual and on the actual situation. Then this process consists in comparing various arguments for and against each decision and in the search for other arguments. During this process one of the variables can increase and another one decrease until the difference between them becomes sufficiently large. At this moment, the decision becomes clear.

In the simplest case, the time evolution of the variables $p_{1}$ and $p_{2}$ can be described by the system of two ordinary differential equations:

$$
\frac{d p_{1}}{d t}=k_{1} p_{1} U_{1}, \quad \frac{d p_{2}}{d t}=k_{2} p_{2} U_{2}
$$

where $U_{1}$ is the level of uncertainty for decision 1 and $U_{2}$ for decision 2 . We will understand uncertainty here not in the same sense as it is usually accepted in the decision theory [3]. Uncertainty for decision 1 is some zone in the intellectual universe of the individual (mind) related to decision 1 , which is not yet conquered by the arguments for or against this decision. We can compare it with resources which can be consumed by the willingness 1 in order to produce more of it. Hence production of the willingness 1 is proportional to its value multiplied by the value of uncertainty for decision 1 . For example, if $U_{1}=0$, there is no uncertainty about decision 1 and, as a consequence, no new possible arguments in favor of this decision or against it, then the willingness 1 will not change. It is similar for the second equation of this system.

At the next stage of the construction of this model, we need to specify the values $U_{1}$ and $U_{2}$. Let $K$ be a measure of the whole intellectual zone related to decisions 1 and 2 . $K$ can be qualified by any of the present theories of uncertainty, like theory of probability, plausibility, possibility, belief, fuzzines, etc [18]. Then we will define the levels of uncertainty as follows:

$$
U_{1}=K-a p_{1}-b p_{2}, \quad U_{2}=K-c p_{1}-d p_{2} .
$$

Hence uncertainty is what remains when we subtract the parts already occupied by decisions 1 and 2. Let us note that the coefficients $a$ and $c, b$ and $d$ can be different. The role of the willingness 1 can be different in the evolution of itself and of the willingness 2 . In the expression for $U_{1}$, the terms $b p_{2}$ shows how willingness 2 acts on the evolution of willingness 1. These are arguments for decision 2 in the evolution of decision 1 . We can interpret it as arguments against decision 1. From this point of view, we have 4 types of arguments, for and against decision 1 in the expression for $U_{1}$, for and against decision 2 in the expression for $U_{2}$.

Thus, we obtain the closed system

$$
\frac{d p_{1}}{d t}=k_{1} p_{1}\left(K-a p_{1}-b p_{2}\right), \quad \frac{d p_{2}}{d t}=k_{2} p_{2}\left(K-c p_{1}-d p_{2}\right) .
$$


Though the analysis of this system is simple and well known, we will briefly repeat it in the next section from the point of view of decision making.

The model described above is related to the probabilistic theory of decision making [18]. Indeed, let $p$ be the probability to take some decision. Then $P=-\ln p$ is a psychophysical variable which can be described in terms of Procrastination. This "abulic-like" variable $P$ decreases with time with the rate proportional to the uncertainty $U$ with respect to this decision: $d P / d t=-k U$. This equation is similar to (2.1) in the case of one decision. Set $U=1-a p$. If $a$ is small, then we get

$$
\frac{d p}{d t} \approx \frac{k e^{-P}}{1+a e^{-P}}
$$

This formula is similar to that of the discrete decision process in the Boltzmann machines [18], where

$$
P(p=1)=\frac{k e^{-P}}{1+a e^{-P}} .
$$

Let us note that system (2.3) is also related to neural models of decision making [20], [21], [22], [23], [24], [25]. Other dynamical systems of decision making are suggested in [26].

\subsection{Mutually exclusive decisions and compromise}

Without loss of generality we can set $K=1$. Otherwise system (2.3) can be easily reduced to this case. Its non-negative stationary points are as follows: $E_{0}=(0,0), E_{1}=(1 / a, 0)$, $E_{2}=(0,1 / d)$ and $E_{3}=\left(p_{1}^{0}, p_{2}^{0}\right)$, where $p_{1}^{0}$ and $p_{2}^{0}$ are solution of the system

$$
a p_{1}+b p_{2}=1, \quad c p_{1}+d p_{2}=1 .
$$

We will suppose that it has a positive solution.

The point $E_{0}$ is always unstable, the points $E_{1}$ and $E_{2}$ are stable if

$$
c>a, \quad b>d .
$$

The point $E_{3}$ is stable if

$$
c<a, \quad b<d \text {. }
$$

The first condition in (2.4) means that the arguments against decision 2 are stronger than the arguments for decision 1. Similarly, the second condition signifies that the arguments against decision 1 are stronger than those for decision 2 . In the other words, the choice is between against decision 1 and against decision 2 . It is what is usually called to choose between two bad decisions. In this case, the choice between them depends on the initial condition.

If conditions (2.5) are satisfied, then arguments for decisions 1 and 2 are stronger than against decisions 2 and 1 . So we choose between two good decisions. However, in this case the intermediate stationary point $E_{3}$ with positive coordinates is stable. So the variables $p_{1}$ 
and $p_{2}$ will converge to it. This stationary point corresponds to the compromise between two decisions. Hence the compromise is possible when we choose between two good options but not possible when we choose between two bad ones.

Further analysis of individual decision making on the basis on neural model dynamical systems can be found in [20], [21], [22], [23].

\subsection{Can two individuals reach agreement?}

The model of individual decision making described above represents a system of two ordinary differential equations with bistable dynamics. In order to study the interaction of two individuals, we will consider here a simplified model of individual decision making which consists of a single equation with the bistable dynamics:

$$
\frac{d q}{d t}=f(q)
$$

where $q$ is the variable which determines individual decision making, $f(q)=a q\left(q-q_{0}\right)(1-q)$, $0<q_{0}<1$. If the initial condition $q(0)$ is greater than $q_{0}$, then the solution will converge to the stationary solution $q=1$. If $q(0)<q_{0}$, then it will converge to the stationary solution $q=0$. These two stable stationary solutions correspond to two decisions between which the individual should make a choice.

Consider two individuals who can influence the decision of each other. Then we obtain the following system of equations for individuals 1 and 2 :

$$
\begin{aligned}
& \frac{d q_{1}}{d t}=f\left(q_{1}\right)+k\left(q_{2}-q_{1}\right), \\
& \frac{d q_{2}}{d t}=f\left(q_{2}\right)+k\left(q_{1}-q_{2}\right) .
\end{aligned}
$$

Here $q_{1}$ is the willingness of individual 1 and $q_{2}$ of individual 2 . The last terms in the right-hand sides of these equations describe the mutual influence of the individuals which is proportional to the difference between their willingness.

Stationary points of this system can be found from the following equations:

$$
q_{2}=-\frac{1}{k} f\left(q_{1}\right)+q_{1}, \quad q_{1}=-\frac{1}{k} f\left(q_{2}\right)+q_{2} .
$$

Let us consider two limiting cases of small and large $k$. If $k$ is sufficiently small, then system (2.7), (2.8) has four stable stationary points with non-negative coordinates. If $k$ is sufficiently large, then this system has only two stable stationary points.

Hence we can conclude that if the mutual influence of the individuals is weak, then four (stable) situations are possible: each of them remains with hir own decision or they have both the same decisions (one of two stable decisions). The choice between these cases depends on the initial values of willingness of the individuals. If their mutual influence is strong, then they will necessaryly have the same decision. Thus, under conditions considered in this section two individuals can reach agreement but not a compromise. 


\subsection{Individual in a hostile environment}

Similar to the previous section, we will consider here a simplified model of individual decision making. Suppose that the individual interacts with hir environment which can influence hir decision. We consider the reaction of the environment and the variable $u$ which measures this reaction. The reaction of the environment is produced as a response to the individual decision making, and it also influences the decision making. We obtain the system of two ordinary differential equations:

$$
\begin{gathered}
\frac{d q}{d t}=a q\left(q-q_{0}\right)(1-q)+k u, \\
\frac{d u}{d t}=b\left(q-q_{0}\right) .
\end{gathered}
$$

Here $a$ and $b$ are some positive constants. The first equation describes individual decision making (cf. equation (2.6)) that is influenced by the environment. The second equation shows the reaction of the environment on decision making. We will suppose that initially the reaction of environment is neutral, $u(0)=0$. It becomes nonzero when the individual is in the process of decision making. It increases in the absolute value when the willingness of the individual goes away from the undecided state $q=q_{0}$. If the coefficient $k$ is positive, then the reaction of environment reinforces the decision of the individual, if $k$ is negative, they work in the opposite directions. We will call this last case the hostile environment because it always acts against the decision of the individual. (see, e.g., [27]).

System (2.10), (2.11) describes the process of decision making in its interaction with active environment. It has a unique stationary point $u=0, q=q_{0}$. If $k<0$, then this stationary point is unstable focus. The solution will oscillate with an increasing amplitude. Hence individual will permanently change hir decision in the hostile environment until other mechanisms will stop these oscillations. This case corresponds to so called unstable "Buridan" decisions [3]. Social dynamics in a hostile environment has various applications including obesity [28], [29] (see also [27]).

\section{Propagation of decision}

\subsection{Model with mutual influence of individuals}

The model of individual decision making will allow us to study the formation or the propagation of decisions in the society. Let us consider a space interval $[0, L]$ where each point corresponds to one individual. We consider a continuous limit of the model with a finite number of individuals. For the individual located at the space point $x$ we introduce the variables $p_{1}(x, t), p_{2}(x, t)$ which characterize hir decision making. These variables are described by a system similar to system (2.3): 


$$
\frac{\partial p_{1}}{\partial t}=f_{1}\left(p_{1}\right)\left(1-a p_{1}-b p_{2}\right)-\gamma_{1} p_{1}, \quad \frac{\partial p_{2}}{\partial t}=f_{2}\left(p_{2}\right)\left(1-c p_{1}-d p_{2}\right)-\gamma_{2} p_{2}
$$

We consider here a more general case where the linear dependencies on $p_{1}$ and $p_{2}$ in the right-hand sides of equations (2.1) are replaced by some functions $f_{1}\left(p_{1}\right)$ and $f_{2}\left(p_{2}\right)$, the last terms describing gradual decay of willingness.

If the individuals can communicate and influence decisions of other individuals, then we need to take this new factor into account. We will consider here the case of mutually exclusive decisions where the points $E_{1}$ and $E_{2}$ are stable and the point $E_{3}$ is unstable. Then we can introduce two types of individuals. The individuals of the first type adopt decision 1 , of the second type decision 2 .

We impose an additional condition which specifies the moment when an individual adopts hir decision. Let $p_{1}^{*}$ be sufficiently close to $1 / a$ (the value at the point $E_{1}$ ) and less than it. Similarly, $p_{2}^{*}$ is close to $1 / d$ and smaller than this value. When the solution $\left(p_{1}(x, t), p_{2}(x, t)\right)$ converges to one of two stable stationary points, it reaches one of the two critical values. If it is $p_{1}^{*}$, then the individual belongs to the first type, if $p_{2}^{*}$, then to the second type. Before the decision is adopted, the type of the individual is not specified (undecided individuals).

Next, we suppose that individuals of type 1 produce some signals. We will denote their level (concentration) by $u_{1}$. Similarly $u_{2}$ denotes the variable which characterizes signals from the individuals of type 2 . These signals should be in relation with decisions 1 and 2 and they can contain any type of visual, oral and other information which can be transmitted between two individuals. We can write the following system of equations for the variables $u_{1}$ and $u_{2}$ :

$$
\begin{aligned}
& \frac{\partial u_{1}}{\partial t}=D_{1} \frac{\partial^{2} u_{1}}{\partial x^{2}}+W_{1}-\sigma_{1} u_{1}, \\
& \frac{\partial u_{2}}{\partial t}=D_{2} \frac{\partial^{2} u_{2}}{\partial x^{2}}+W_{2}-\sigma_{2} u_{2},
\end{aligned}
$$

where $W_{1}$ and $W_{2}$ are the rates of production of the corresponding signals, the last terms describing their decay. Diffusion terms in these equations describe diffusion of signals (information). Similar to the usual molecular diffusion, the flux of information is proportional to the difference of the signal level between the individuals who exchange information.

The variables $u_{1}$ and $u_{2}$ influence individual decision making:

$$
\begin{aligned}
& \frac{\partial p_{1}}{\partial t}=f_{1}\left(p_{1}\right)\left(1-a p_{1}-b p_{2}\right)-\gamma_{1} p_{1}+b_{11} u_{1}+b_{12} u_{2}, \\
& \frac{\partial p_{2}}{\partial t}=f_{2}\left(p_{2}\right)\left(1-c p_{1}-d p_{2}\right)-\gamma_{2} p_{2}+b_{21} u_{1}+b_{22} u_{2} .
\end{aligned}
$$

Let us note that in Section 2.3, where we consider the mutual influence of two individuals, they interacted directly through their willingness. The model of interaction by intermediate of produced signals is more general. 
It remain to specify how the rates of production of the signals $u_{1}$ and $u_{2}$, respectively $W_{1}$ and $W_{2}$, depend on individual decision making. Consider a space point $x$ and denote by $t_{0}$ the minimal time for which $p_{1}\left(x, t_{0}\right)=p_{1}^{*}$ or $p_{2}\left(x, t_{0}\right)=p_{2}^{*}$. In the first case we set

$$
c_{1}(x, t)=\left\{\begin{array}{ll}
0, & t<t_{0} \\
1, & t \geq t_{0}
\end{array} \quad, \quad c_{2}(x, t)=0,\right.
$$

while in the second case

$$
c_{2}(x, t)=\left\{\begin{array}{ll}
0, & t<t_{0} \\
1, & t \geq t_{0}
\end{array} \quad, \quad c_{1}(x, t)=0 .\right.
$$

We can now define $W_{1}$ and $W_{2}$ :

$$
W_{1}=\alpha_{1} c_{1}, \quad W_{2}=\alpha_{2} c_{2}
$$

with some $\alpha_{1}, \alpha_{2}>0$. Thus, if $p_{1}$ reaches the critical value $p_{1}^{*}$ and $p_{2}<p_{2}^{*}$, the undecided individual adopts the first decision. After that he begins to produce signal $u_{1}$. It occurs similarly with the second decision.

We will consider the functions $f_{1}(p)$ and $f_{2}(p)$ in the following form:

$$
f_{1}(p)=k_{1} p+n_{1} p^{2}, \quad f_{2}(p)=k_{2} p+n_{2} p^{2},
$$

where $k_{1}, k_{2}, n_{1}, n_{2}$ are non-negative numbers. The case of linear functions corresponds to the usual model of competition of species. We will see that behavior of solutions can be essentially different for the quadratic dependence.

System (3.2)-(3.5) is a degenerate parabolic system with some of the diffusion coefficients equal zero. Travelling wave solutions for some classes of degenerate parabolic systems are studied in [30], [31], [32]. We will discuss below the modes of propagation of such waves. We will observe propagation of simple waves with a constant solution behind the wave front and periodic waves with a spatial structure behind the wave (cf. [33]).

In the next sections we present the results of numerical simulations of this model. We used an implicit finite difference approximation of the diffusion operator with Thomas algorithm to inverse the tridiagonal matrix. The nonlinear terms were taken explicitly (from the previous time step). Accuracy of the simulations was verified by decreasing the space and time steps. Let us note that this is a qualitative model, and the values of parameters are chosen to illustrate different regimes of wave propagation. We will use the following values:

$$
\begin{gathered}
a=0.5, b=1, c=1, d=0.5, \alpha_{1}=\alpha_{2}=0.1, \gamma_{1}=\gamma_{2}=0.1, \sigma_{1}=\sigma_{2}=0.1, \\
D_{1}=D_{2}=0.1, p_{1}^{*}=p_{2}^{*}=1.5
\end{gathered}
$$

unless other values are indicated. The values of other parameters will be given in the corresponding simulations. 


\subsection{Do as the others}

Behavior of solutions of this problem depends on the values of the coefficients. We begin with the case where $b_{12}=b_{21}=0, b_{11}$ and $b_{22}$ are positive. Therefore the signal $u_{1}$, produced by individuals adopting decision 1 , acts to reinforce decision 1 . Similar for the signal $u_{2}$. We can interpret this case as doing similar to the others.

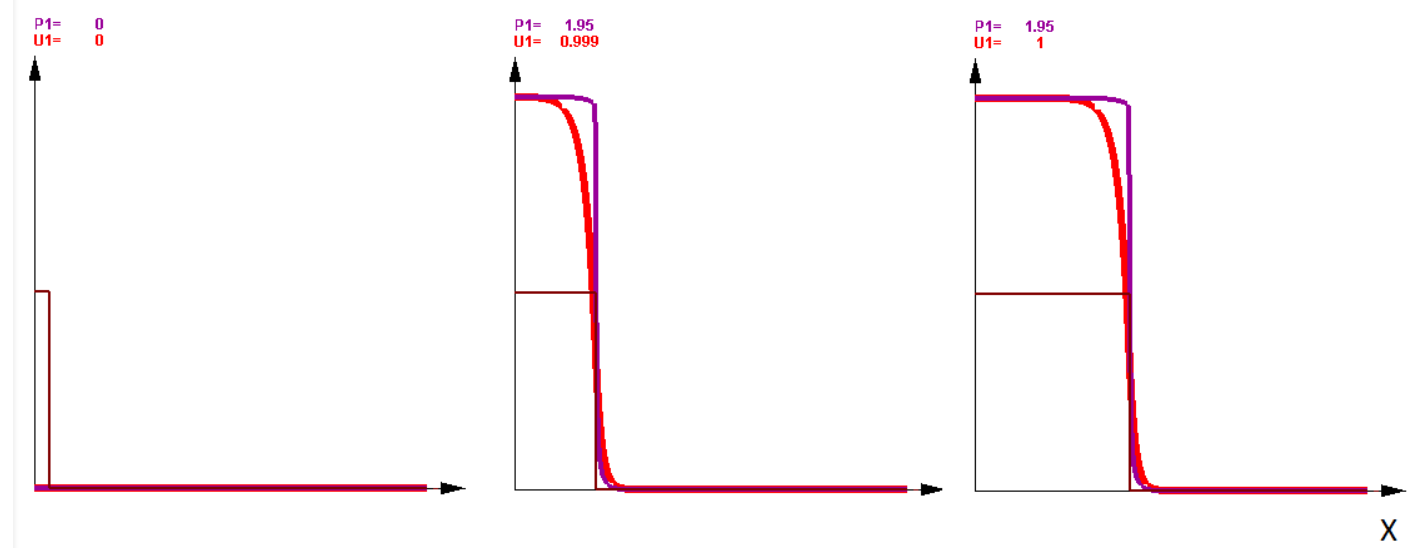

Figure 1: Snapshots of solution in three consecutive moments of time. Individuals of type 1 gradually fill the whole interval ( $c_{1}=1$, black curves). Their propagation is accompanied by propagation of signal $u_{1}$ (red curves) and of the internal variable $p_{1}$ (violet curves). The values of parameters are as follows: $k_{1}=k_{2}=0.5, n_{1}=n_{2}=0 ; b_{11}=0.1, b_{12}=0, b_{21}=0, b_{22}=0.1$

Let the initial distributions for $p_{1}, p_{2}, u_{1}$ and $u_{2}$ be identically zero. We introduce at the left border of the interval individuals $1\left(c_{1}=1\right)$. They produce signal $u_{1}$ which diffuse, initiate production of the variable $p_{1}$ at the individual decision level. When this variable reaches the critical level, the undecided individuals become individuals of type 1 . They produce signal $u_{1}$ in their turn and so on. We observe propagation of a front wave where individuals 1 gradually fill the whole interval (Figure 1). Individuals of type 2 do not appear and the corresponding variables $p_{2}$ and $u_{2}$ remain zero. This is a reaction-diffusion wave similar to those observed in various models of population dynamics [32].

We recall that the evolution of willingness in equations $(3.4),(3.5)$ is determined by its production, by its decay (or dissipation) and by the external influence. If the production of willingness is not fast enough to compensate its dissipation, then the critical value $p_{1}^{*}$, which is necessary for the undecided individuals to adopt decision 1, may not be reached. Then we observe a strange effect where the interval remains filled by the undecided individuals but their willingness rises from 0 to some constant value (Figure 2, left).

We can give the following explanation of this behavior. Individuals of type 1 introduced initially produce signal $u_{1}$ which propagates and converges to an exponentially decaying distribution. In spite of its fast decay, it is sufficient to stimulate production of willingness $p_{1}$ everywhere in space. However, since the signal decays, the individuals located at greater 

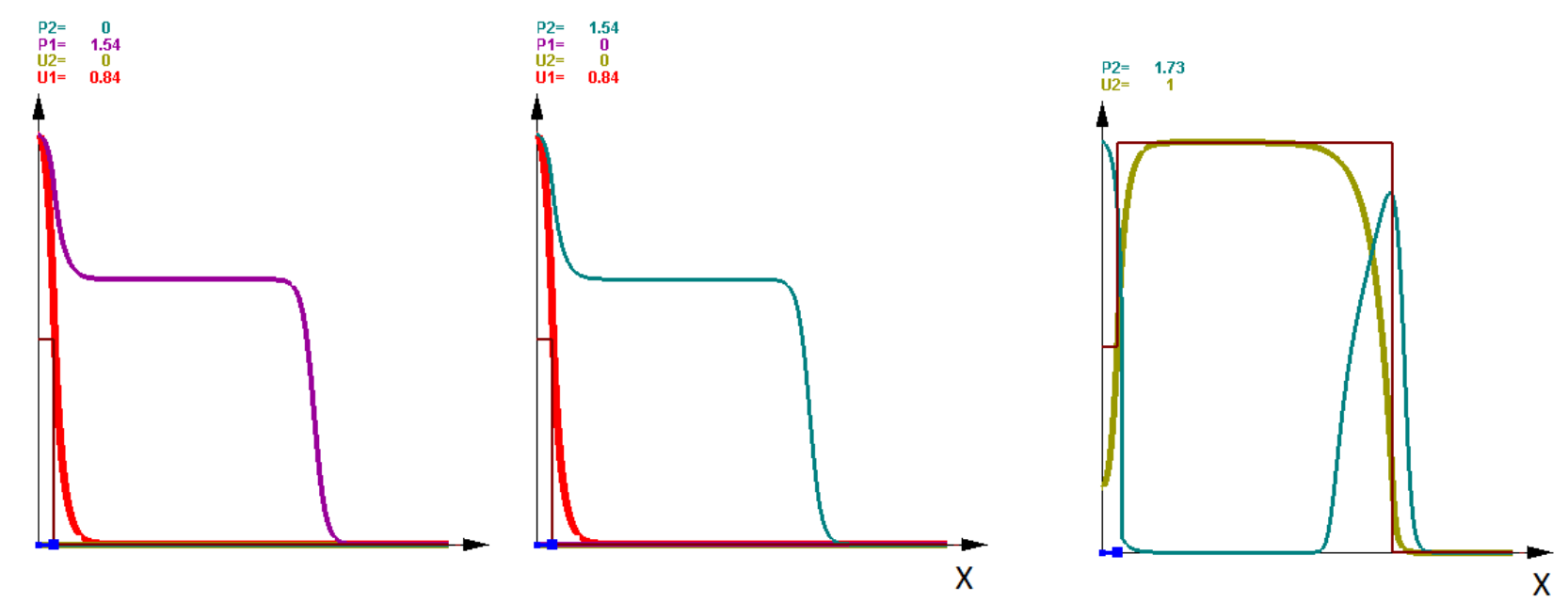

Figure 2: Propagation of willingness in the case of doing as the others, $k_{1}=k_{2}=0.2$, $n_{1}=n_{2}=0 ; b_{11}=0.1, b_{12}=0, b_{21}=0, b_{22}=0.1$ (left), and in the case of doing opposite to the others $b_{11}=0, b_{12}=0.1, b_{21}=0.1, b_{22}=0$ (middle). If production of willingness is faster, then individuals of type 2 appear and spread in space, $k_{1}=k_{2}=0.5, n_{1}=n_{2}=0$; $b_{11}=0, b_{12}=0.1, b_{21}=0.1, b_{22}=0$ (right). The colors of the curves correspond to the colors of the variables above the figures.

distance need more time to develop their willingness. Its value is not sufficient to adopt decision 1, and new individuals of type 1 do not appear. Formally, it looks like propagation of the wave of willingness. It is important to note here that this effect is possible because undecided individuals are unstable. In other words, the stationary point $E_{00}$ is unstable, and even a weak external signal leads to the growth of willingness.

This situation can be compared with propagation of rumors where people have not seen the event (here, individuals of type 1) but they form an opinion about this event. This opinion is not strong enough to adopt the corresponding decision.

Finally, if the coefficients $k_{1}$ and $k_{2}$ are sufficiently small, then the rate of production of willingness becomes even less than before, and the willingness will not propagate.

\subsection{Do opposite to the others}

In this section we will consider the case where $b_{11}=b_{22}=0$ and $b_{12}, b_{21}$ are positive. This means that the signal produced by individuals 1 stimulate decision 2 and vice versa. From the point of view of individual decision making, we will interpret this case as doing the opposite to the others. We will see that there are two different subcases here, with unstably and stably undecided individuals. 


\subsubsection{Unstably undecided individuals}

We will begin with the case where the stationary point $E_{00}$ of the individual decision making system is unstable. We set $n_{1}=n_{2}=0$ and $k_{1}, k_{2}$ are positive. Figure 2 (middle) shows behavior similar to that described above where individuals of type one do not expand but provide propagation of the willingness. In this case it is $p_{2}$ instead of $p_{1}$ because individuals of type 1 stimulate production of willingness 2 .

If we increase coefficients $k_{1}$ and $k_{2}$, then willingness 2 reaches the critical value, and individuals 2 are formed and expand in space (Figure 2, right). Hence, introduction of individuals of type 1 leads to propagation of individuals of type 2 . For all combinations of parameters considered here, one of the individual types rapidly dominates another one and propagates, filling the whole space interval.

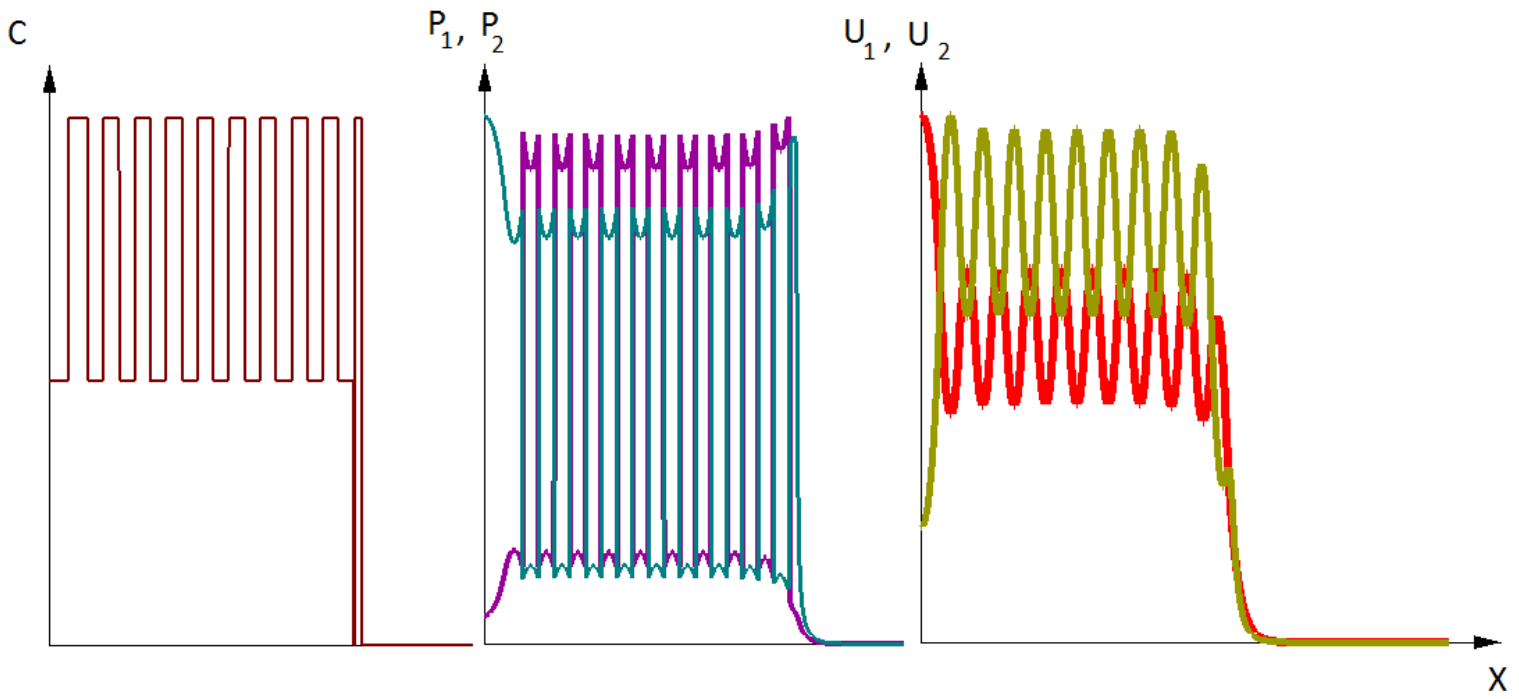

Figure 3: In the case of doing opposite to the others and stably undecided individuals, a periodic pattern appears behind the propagating wave. Both types of individuals emerge and form clusters. A snapshot of solution: distributions of $C$ (left), of $p_{1}, p_{2}$ (middle) and of $u_{1}, u_{2}$ (right).

\subsubsection{Stably undecided individuals}

Another situation is observed if the stationary point $E_{00}$ is stable. We now set $k_{1}=k_{2}=0$ and $n_{1}, n_{2}$ are positive. Since the production terms are quadratic for small $p_{i}$ and decay terms are linear, then the point is stable. Let us note that this case is also realistic because individuals can possess some stability with respect to the external influence on their decision.

We will present an example of numerical simulations where $b_{11}=b_{22}=0$ and $b_{12}, b_{21}$ are positive. This means that individuals of type 1 produce signal which stimulates decision 2 

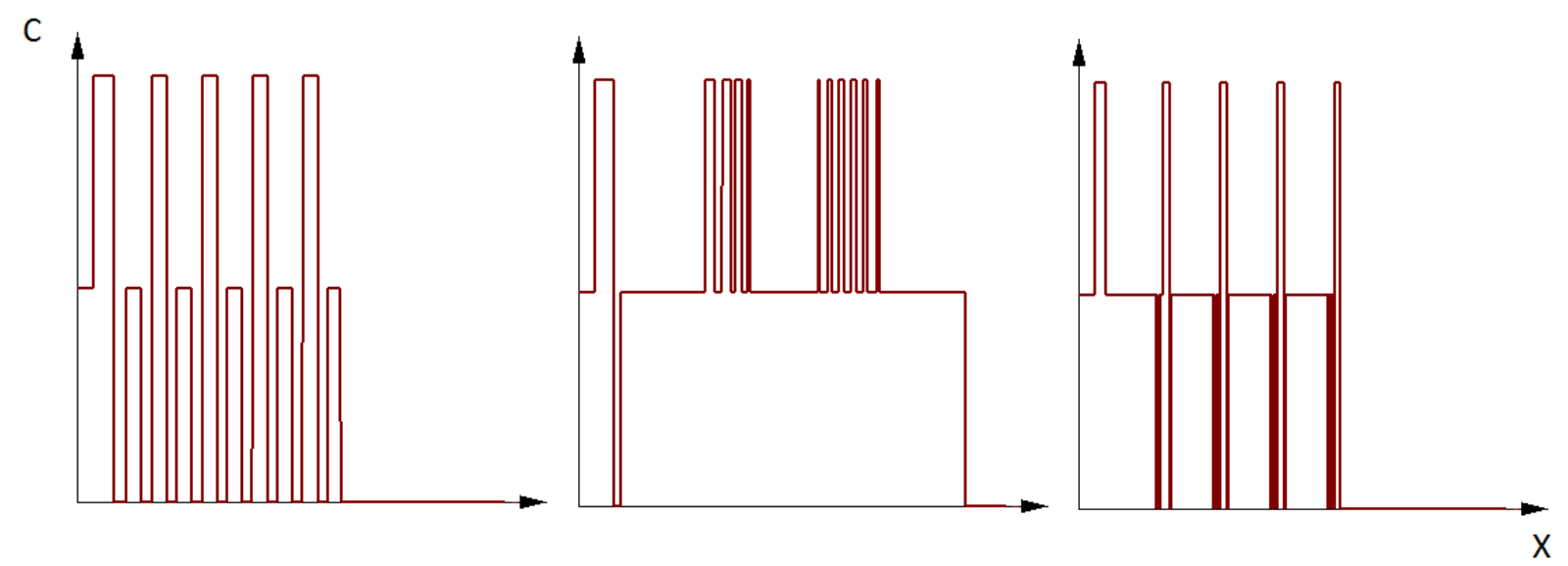

Figure 4: Distribution of individuals behind the wave depends on parameters. It can contain individuals of types 1 and 2 and also undecided individuals; $q_{1}=q_{2}=0.01$ (left), $q_{1}=q_{2}=$ $0.01, D_{2}=0.5$ (middle), $q_{1}=0.1, q_{2}=0.01$ (right).

and individuals of type 2 produce signal which stimulates decision 1 (Figure 3 ). Initially all individuals are undecided $\left(p_{1}=p_{2}=0\right)$. We introduce individuals of type 1 at the left side of the interval and observe a wave propagating from the left to the right. There is a periodic structure behind the wave. Let us note that Figure 3 (left) shows the variable $C$ which takes three values: 0 if $c_{1}=c_{2}=0,1$ if $c_{1}=1, c_{2}=0,2$ if $c_{1}=0, c_{2}=1$. Thus we observe formation of clusters of individuals of types 1 and 2 .

For other values of parameters the propagating patterns can be different. They can contain both types of individuals together with undecided individuals (Figure 4, left). Moreover their distribution depends on parameters (cf. Figure 4, right). Figure 4 (middle) shows a pattern with two types of individuals which is different in comparison with Figure 3.

\section{Discussion}

The results of the work can help to understand certain aspects of social behavior in their relation with the mechanisms of decision making. In particular, we formulate the conditions of the coexistence of different decisions in the society. It has crucial importance in the democratic societies. Moreover, we identify the relation between different types of social behavior and individual neural networks.

Another possible application concerns expert systems. The anticipation in artificial intelligence, notably in medical decision making, requires a consensus between several actors (physician, patient, paramedics) and one of the reasons for the failure of medical expert systems is probably the lack of the modeling of the consensus process among the various players in the medical decision. This process could be revisited with the quantification proposed in this article, with a modeling of the mutual influences between health strategy makers.

Individual decision making is a choice between two or several options. This choice can 
be influenced by many external factors, and by other individuals with their opinions, for example, appearance, public opinion, education, mass media and even physical conditions of the individual. All these external factors can reinforce the decision of the individual or act in the opposite way. Trying to follow these factors, the individual can change hir decision. As in the example with weight loss, these changes can be repetitive.

Another aspect of the same question is whether the individual will follow the external signal or will act contrary to it. The education process is mainly based on following some other's example or pattern of behavior. These can be parents, tutors, friends. A similar mechanism works for young animals who learn from their parents following them.

However, young children at certain age go further, they learn to do opposite. Once they understand that they can refuse doing something, during some period of time they answer "no" to everything. After some time this stage of learning by opposition is finished and their behavior becomes more complex combining positive and negative decisions. The next period of learning by opposition is teenager crisis. Learning by opposition can be an important property of human mind. New ways are found by those who refuse to follow the old ones.

The results presented above show that individual making decision in opposition to received signals can play an important role in the pattern of decisions. They make the decision distribution possibly heterogeneous. This is related to the model of individual in a hostile environment. In the other case, if individuals only follow received signals, there is always one decision dominating the other one (in the framework of the considered models).

Preservation of plurality in decisions is of course very important, in particular for the existence of modern democracy which is based on the equilibrium of two opposing (though not necessarily opposite) forces. There are many examples of coexistence of opposite decisions in the society, such as smoking, obesity, and so on. If individuals with different decisions already exist, then they can form clusters on the basis of their similarity [29]. However these different groups will persist if individuals can oppose to suggested pattern and not only follow it. Otherwise one of them will dominate and suppress another one. Propagation of obesity is studied in [28]. If the individuals can change their decisions due to mutual interactions, then the system can converge to a single equilibrium point where the two decisions coexist [26].

Let us finish this work with the citation from Condorcet about individuals following the opinion of the others: "Celui qui finit par se ranger au parti le plus nombreux, parle certainement contre sa conscience. Puis donc qu'il ne faut pas que l'opiniâtreté d'un seul juge, qui peut être gagné, interrompe le cours de la justice, il ne faut pas faire une loi de l'unanimité" [34]. Thus, he wrote, we should not follow the majority against our convictions. Unanimity in the decision should not make the law.

\section{References}

[1] T. Connolly, H.R. Arkes, K.R. Hammond. Editors. Judgment and decision making. Cambridge University Press, 2000. 
[2] D. Murphy, D. Longo. Editors. Encyclopedia of psychology of decision making. Nova Science Publishers, 2009.

[3] S.O. Hansson. Decision theory. A brief introduction. Uppsala University, 1994.

[4] N. Condorcet. Plan de Constitution, présenté à la convention nationale les 15 et 16 février 1793. Oeuvres, O'Connor and M. F.Arago, éds, Firmin Didot Frères, Paris, 12 (1847), pp. 333415.

[5] J. Dewey. How we think. Southern Illinois University Press, Vol. 6, 1978.

[6] H. Simon. The new science of management decision, Harper and Row, 1960.

[7] O.G. Brim. Personality and decision processes. Studies in the social psychology of thinking. Stanford, 1962.

[8] H. Mintzberg, D. Raisinghani, A. Théorêt. The structure of unstructured decision processes. Administrative Sciences Quarterly, 21 (1976), 246-275.

[9] P.C. Fishburn. Utility theory for decision making. John Wiley \& Sons, 1970.

[10] E.R. Alexander. The limits of uncertainty: a note. Theory and Decision, 6 (1970), 363-370.

[11] D. Hardman, L. Macchi. Editors. Thinking: psychological perspectives on reasoning, judgment and decision making. John Wiley \& Sons, 2003.

[12] P. Hammerstein, J.R. Stevens. Editors. Evolution and the mechanisms of decision making. Strungmann Forum Reports, 2012.

[13] G. Goertz. Constraints, compromises, and decision making. Journal of Conflict Resolution, 48 (2004), 14-37.

[14] T.V. Guy, M. Karny, D.H. Wolpert. Editors. Decision making with imperfect decision makers. Springer, 2012.

[15] A. Kelly. Decision making using game theory: an introduction for managers. Cambridge University Press, 2003.

[16] R. Soeiro, A. Mousa, T.R. Oliveira, A.A. Pinto. Dynamics of human decisions. Journal of Dynamics and Games, 1 (2014), no. 1, 121-151.

[17] J. Demongeot, C. Robert. A comparison between different uncertainty coefficients used in artificial intelligence. In: Artificial Intelligence \& Cognitive Sciences, Manchester Un. Press, Manchester, 1988, pp. 177-186.

[18] E.H. Shortliffe, B.G. Buchanan. A model of inexact reasoning in medicine. Mathematical Biosciences, 23 (1978), 351-379. 
[19] V. V. Kolbin. Decision making and programming. World Scientific Publishing, 2003.

[20] A. Banerjee. Initializing neural networks using decision trees. In: Computational learning theory and natural learning systems. MIT Press Cambridge, MA, USA, 4 (1997), $3-15$.

[21] G.M. Barnwell. A mathematical model of decision-making neural circuits controlling behavioral mode selection. Bulletin of Math. Biology, 39 (1977), 223-237.

[22] J. R. Busemeyer, R. K. Jessup, J. G. Johnson, J. T. Townsend. Building bridges between neural models and complex decision making behaviour. Neural Networks, 19 (2006), 1047-1058.

[23] J. I. Gold, M. N. Shadlen. The Neural Basis of Decision Making. Annu. Rev. Neurosci., 30 (2007), 535-574.

[24] J.J. Hopfield. Neural networks and physical systems with emergent collective computational abilities. Proc. Natl. Acad. Sci., 79 (1982), 2554-2558.

[25] T. Kohonen. Self-organized formation of topologically correct feature maps. Biological Cybernetics 43 (1982), 59-69.

[26] E. Venturino. Does democracy really work? International Journal of Applied Science and Appplications, 8 (2001), 71-93.

[27] A.K. Walker, I.E. Ibia, J.M. Zigman. Disruption of cue-potentiated feeding in mice with blocked ghrelin signaling. Physiol. Behav., 108 (2012), 34-43.

[28] C. Taramasco. Impact de lobésité sur les structures sociales et impact des structures sociales sur l'obésité. PhD thesis, 2011.

[29] C. Taramasco, J. Demongeot. Collective intelligence, social networks and propagation of a social disease, obesity. IEEE Proceedings, Piscataway, 2011, pp. 86-90.

[30] B. Kazmierczak, V. Volpert. Travelling calcium waves in a system with non-diffusing buffers. M3AS, 18 (2008), no. 5, 883-912.

[31] B. Kazmierczak, V. Volpert. Calcium waves in a system with immobible buffers as a limit of waves with a system with nonzero diffusion. Nonlinearity, 21 (2008), No. 1, 71-96.

[32] V. Volpert. Elliptic partial differential equations. Volume 2. Reaction-diffusion equations. Birkhäuser, 2014.

[33] V. Volpert, V. Vougalter. Emergence and propagation of patterns in nonlocal reactiondiffusion equations arising in the theory of speciation. In: "Dispersal, individual movement and spatial ecology", Eds. M. Lewis, Ph. Maini, S. Petrovskii. Springer Applied Interdisciplinary Mathematics Series, 2013, 331-353. 
[34] N. Condorcet. Correspondance avec Turgot sur les jurys. In: Condorcet, Arithmétique politique. Textes rares ou inédits (1767-1789), B. Bru et P. Crépel, éds, INED, Paris (1994), p. 60. 